\title{
Pattern reconstruction and sequence processing in feed-forward layered neural networks near saturation
}

\author{
F. L. Metz and W. K. Theumann \\ Instituto de Física, Universidade Federal do Rio Grande do Sul, Caixa Postal 15051, 91501-970 Porto Alegre, Brazil
}

(Received 3 December 2004; published 23 August 2005)

\begin{abstract}
The dynamics and the stationary states for the competition between pattern reconstruction and asymmetric sequence processing are studied here in an exactly solvable feed-forward layered neural network model of binary units and patterns near saturation. Earlier work by Coolen and Sherrington on a parallel dynamics far from saturation is extended here to account for finite stochastic noise due to a Hebbian and a sequential learning rule. Phase diagrams are obtained with stationary states and quasiperiodic nonstationary solutions. The relevant dependence of these diagrams and of the quasiperiodic solutions on the stochastic noise and on initial inputs for the overlaps is explicitly discussed.
\end{abstract}

DOI: 10.1103/PhysRevE.72.021908

PACS number(s): 87.10.+e, 64.60.Cn, 07.05.Mh

\section{INTRODUCTION}

Models of attractor neural networks for processing sequences of patterns, as a realization of a temporal association, have been of great interest following Hopfield's pioneering work [1-7] and renewed interest has come through both the availability of new theoretical dynamic approaches to study the evolution of disordered systems, in particular, neural networks near saturation [8-12] and experimental findings. Among the latter are the results of Miyashita et al., who showed that serial positions of stimuli to which monkeys were exposed during training are converted to spatial correlations of neural activities $[13,14]$.

A neural network model with a symmetric learning rule that consists of a Hebbian part and a pair of pattern sequences has been proposed to interpret the experiments $[15,16]$. The analysis of the equilibrium states for both finite and extensive loading of patterns revealed the presence of correlated attractors with a decreasing correlation as the separation of the patterns in the sequence increases, in apparent support of the experimental work. Phase diagrams were obtained where correlated attractors compete with Hopfieldlike attractors and with symmetric states $[17,18]$. The dynamical evolution to the stationary states has been studied recently by means of dynamical replica theory [19].

Neural network models for sequence processing with asymmetric interactions are more natural from a biological point of view. Due to the lack of microscopic detailed balance, however, the equilibrium states of the network cannot be obtained by means of the statistical mechanics approach [20], and one has to resort to a dynamical study. Analytic and numerical studies of the stationary states and some aspects of the dynamics for the competition between pattern reconstruction and asymmetric sequence processing in the case of finite loading of patterns, that is, in the absence of stochastic noise, where the ratio $\alpha=p / N$ between the number of patterns $p$ and units $N$ is zero, appeared in works by Coolen et al. $[21,22]$. Phase diagrams for the stationary states of a parallel dynamics exhibit either stable Hopfield-like or symmetric mixture states characterized by fixed-point solutions and stable limit cycles described by periodic fixed points. Non- stationary solutions of the dynamics also appear for an increasingly large number of patterns [21]. More recent works deal with stationary limit cycles in asymmetric sequence processing without pattern reconstruction, for extensive loading of patterns [12,23-26].

It is important to consider the effects of the extensive loading of patterns and to study the stability of the states that appear in the competition between pattern reconstruction and asymmetric sequence processing to the presence of stochastic noise (finite $\alpha$ ). This noise could be due to the presence of a previously learned macroscopic number of patterns following a specific learning rule. Since the problem is a dynamical one, it is also important to study the dependence of the network performance on initial conditions.

Those are issues that, apparently, have not been considered before and the purpose of the present paper is to study them on a tractable dynamical feed-forward layered neural network model with no feedback loops [27]. This is an extensively used model that consists of identical layers of $N$ noninteracting units on each layer, with synaptic interactions only between units on consecutive layers. The feed-forward nature of the model and the updating of the units on each layer endow the network of a dynamics in which the layer index becomes a discrete time. We consider an interaction matrix that yields either a static Hebbian noise or a dynamic Hebbian plus-sequential noise and restrict the work to binary units and patterns. In the finite loading case, the model becomes identical to that of Coolen and Sherrington for a parallel dynamics [21].

The outline of the paper is the following. In Sec. II we present the model and the relevant order parameters. In Sec. III we derive the macroscopic dynamics of the network and in Sec. IV we present the results in the form of phase diagrams of stationary states and regions of nonstationary solutions. We end with a discussion and conclusions in Sec. V.

\section{THE MODEL}

The network model consists of $L$ layers with $N$ binary Ising units (neurons) on each layer $l$ in a microscopic state $\boldsymbol{\sigma}(l)=\left\{\sigma_{1}(l), \ldots, \sigma_{N}(l)\right\}$, in which each $\sigma_{i}(l)= \pm 1$. The state 
+1 represents a firing neuron and the state -1 a neuron at rest. The microscopic dynamics of the network is generated as follows: given a configuration on the first layer, $\boldsymbol{\sigma}(1)$, all units on layer $l+1$ are updated simultaneously according to the alignment of each unit $i$ to its local field,

$$
h_{i}(l+1)=\sum_{j=1}^{N} J_{i j}(l) \sigma_{j}(l)
$$

due to the states of the units on the previous layer $l$, following a stochastic law with probability

$$
\operatorname{Prob}\left(\sigma_{i}(l+1) \mid \boldsymbol{\sigma}(l)=\frac{\exp \left[\beta \sigma_{i}(l+1) h_{i}(l+1)\right]}{2 \cosh \left[\beta h_{i}(l+1)\right]} .\right.
$$

Thus, the network has a parallel dynamics with no feedback loops in which the layer indices may be associated with discrete time steps. Here, $J_{i j}(l)$ is the synaptic connection defined below between unit $j$ on layer $l$ and unit $i$ on layer $l$ +1 . The parameter $\beta=T^{-1}$ controls the synaptic noise such that the dynamics of the network becomes a deterministic one when $T \rightarrow 0$ and fully random when $T \rightarrow \infty$. In the former case, the state of a unit is given by the deterministic form

$$
\sigma_{i}(l+1)=\operatorname{sgn}\left[h_{i}(l+1)\right] .
$$

A macroscopic set of $p=\alpha N$ independent and identically distributed random patterns $\xi^{\mu}(l)=\left\{\xi_{1}^{\mu}(l), \ldots, \xi_{N}^{\mu}(l)\right\}, \mu$ $=1, \ldots, p$, and each $\xi_{i}^{\mu}(l)= \pm 1$ with probability $\frac{1}{2}$ are stored in the learning stage on the units of each layer $l$, independently of other layers. Since we are interested in the retrieval of one or a small number $c$ of patterns and in the recognition of a finite sequence, we assume a learning rule of the form

$$
J_{i j}(l)=\frac{1}{N} \sum_{\mu, \rho=1}^{c} \xi_{i}^{\mu}(l+1) A_{\mu \rho} \xi_{j}^{\rho}(l)+\frac{1}{N} \sum_{\mu, \rho=c+1}^{p} \xi_{i}^{\mu}(l+1) B_{\mu \rho} \xi_{j}^{\rho}(l),
$$

where

$$
\begin{aligned}
& A_{\mu \rho}=\nu \delta_{\mu \rho}+(1-\nu) S_{\mu \rho}, \quad \mu \bmod c \\
& B_{\mu \rho}=b \delta_{\mu \rho}+(1-b) S_{\mu \rho}, \quad \mu \bmod p
\end{aligned}
$$

in which $B_{\mu \rho}$ is nonzero only for $\mu \geqslant c+1$. These are the (layer-independent) elements of matrices $\mathbf{A}$ and $\mathbf{B}$, with continuous independent $\nu$ and $b(0 \leqslant \nu, b \leqslant 1)$, in which $\delta_{\mu \rho}=1$ if $\mu=\rho$, and zero otherwise, while $S_{\mu \rho}=\delta_{\mu, \rho+1}$ is a permutation matrix element. The signal-to-noise analysis carried out later depends on the assumption that the patterns can be separated into $c$ "low" or condensed ones, with finite overlaps with the states of the network in the $N \rightarrow \infty$ limit and $(p-c)$ "high" or noncondensed patterns with overlaps of $O(1 / \sqrt{N})$ with the states. The assumption is justified in the case of a blockmatrix interaction (here, a diagonal two-block matrix) and the specific form we choose is inspired in an earlier proposal [18]. In that spirit we assume here not necessarily the same weights, $\nu$ and $b$. This is in order to contemplate different simple possibilities and to avoid an eventual closure problem in the noise term discussed below. The first part of $J_{i j}(l)$ represents a finite cycle that is a superposition of a Hebbian learning (the diagonal part of $\mathbf{A}$ ) of single patterns and of patterns in a sequence (the nondiagonal part). Again, in the spirit of that reference, one may think of the condensed part in the presence of an infinite cycle (in the large- $p$ limit) of a previously learned similar superposition of Hebbian and sequential patterns represented by the second part of $J_{i j}(l)$, which acts as a noise. Eventually, one could have $0 \leqslant \nu \leqslant 1$ and a Hebbian noise with $b=1$ [18], which is a rather convenient choice.

The learning stage of the network is a dynamical process that involves patterns on two consecutive layers. The Hebbian part of the rule may be thought as a static process that reinforces the learning of the same pattern on every pair of consecutive layers (times), whereas the sequential part of the rule is a dynamic process in which the synaptic interaction is due to a pattern at a given time with the following pattern in the sequence at the next discrete time. It has been found before, in studying the finite loading case, that the competing static process has a stabilizing effect on the dynamic sequential process leading to a phase of symmetric states, which are the only ordered stable states within a wide range of relative synaptic strengths under an appropriate amount of synaptic noise parameter $T$. Eventually, the static process may fail to lock the transitions in the dynamic process and nonstationary quasiperiodic solutions may appear that have already been found in the case of finite loading [21]. These are natural features of the model that are enhanced by stochastic noise (finite $\alpha$ ), as will be seen and discussed in this work.

The properties of $\mathbf{A}$, in particular its eigenvalues and the complete set of orthogonal eigenvectors, lead to symmetries of the solutions of the nonlinear parallel dynamics in the form of a $\nu /(1-\nu)$ duality, that has been discussed for a finite loading of patterns [21]. Here we find an additional $b /(1-b)$ duality. For the case of the continuous bifurcations of solutions, the eigenvalues of the matrix yield the transition temperatures to the ordered states and the eigenvectors give the symmetry directions of the macroscopic overlaps between the states of the network and the patterns.

We define the macroscopic overlap between the configuration $\boldsymbol{\sigma}(l)$ of the network on layer $l$ and one or more condensed key patterns $\boldsymbol{\xi}^{\mu}(l), \mu=1, \ldots, c$, on that layer as the large- $N$ limit, $m^{\mu}(l)$, of

$$
m_{N}^{\mu}(l)=\frac{1}{N} \sum_{i=1}^{N} \xi_{i}^{\mu}(l)\left\langle\sigma_{i}(l)\right\rangle,
$$

where the brackets denote a thermal average with Eq. (2). Since the number of condensed patterns is finite, one may use the self-averaging property to write $m^{\mu}(l)=\left\langle\xi_{i}^{\mu}(l)\right.$ $\left.\times\left\langle\sigma_{i}(l)\right\rangle\right\rangle$, where the outer bracket here and below denotes a configurational average over the patterns. Similarly, we define the overlap between the same configuration and a given noncondensed pattern $\xi^{\mu}(l), \mu=c+1, \ldots, p$, on layer $l$,

$$
M_{N}^{\mu}(l)=\frac{1}{N} \sum_{i=1}^{N} \xi_{i}^{\mu}(l) \sigma_{i}(l) .
$$

Assuming that a given configuration of the first layer has a finite overlap $m^{\mu}(1)=O(1)$ with one or more condensed pat- 
terns and overlaps $M_{N}^{\mu}(1)=O(1 / \sqrt{N})$ with the noncondensed patterns, the dynamic evolution of the network will yield overlaps $m^{\mu}(l)=O(1)$ and $M_{N}^{\mu}(l)=O(1 / \sqrt{N})$ on the following layers. We consider next the evolution equations for the overlaps.

\section{DYNAMICS OF THE NETWORK}

Adapting the standard procedure for the layered network to our model, we write the local field at a unit on layer $l$ +1 due to the overlaps with all patterns on layer $l$, in the large- $N$ limit [27],

$$
h_{i}(l+1)=\sum_{\mu, \rho=1}^{c} \xi_{i}^{\mu}(l+1) A_{\mu \rho} m^{\rho}(l)+z_{i}(l),
$$

where the first term is the signal and $z_{i}(l)$ is the large- $N$ limit of the noise

$$
R_{i}(l)=\sum_{\mu, \rho=c+1}^{p} \xi_{i}^{\mu}(l+1) B_{\mu \rho} M_{N}^{\rho}(l),
$$

due to the overlaps of the states with the noncondensed patterns. This is a random quantity in both the patterns on layer $l+1$ and the implicit dependence on thermal and configurational randomness of the overlaps $M_{N}^{\mu}(l)$ on layer $l$ due to the previous layers.

The noise gives a finite contribution to the local field. Indeed, due to the fact that $M_{N}^{\mu}(l)=O(1 / \sqrt{N})$ and that $R_{i}(l)$ is a sum of a large number of statistically independent random variables, one can first apply the central limit theorem and then the law of large numbers to conclude that $z_{i}(l)$ follows a Gaussian distribution with mean zero and a variance $\Delta^{2}(l)$ given by the large- $N$ limit of

$$
\Delta_{N}^{2}(l)=\sum_{\mu=c+1}^{p}\left\langle\left\langle\left[b M_{N}^{\mu}(l)+(1-b) M_{N}^{\mu-1}(l)\right]^{2}\right\rangle\right\rangle .
$$

We used the fact that the patterns are unbiased and uncorrelated random variables with $\left\langle\xi_{i}^{\mu}(l+1)\right\rangle=0$ and $\left\langle\xi_{i}^{\mu}(l+1) \xi_{i}^{\nu}(l\right.$ $+1)\rangle=\delta_{\mu \nu}$.

We make use now of the local field to derive the recursion relations for the vector overlaps with the condensed patterns, $\boldsymbol{m}(l)=\left\{m^{\mu}(l)\right\}, \mu=1, \ldots, c$, and for the variance of the noise. For the former we obtain

$$
\boldsymbol{m}(l+1)=\left\langle\boldsymbol{\xi}(l+1) \int D z \tanh \{\beta[\boldsymbol{\xi}(l+1) \cdot \boldsymbol{A} \boldsymbol{m}(l)+\Delta(l) z]\}\right\rangle,
$$

where $D z=e^{-z^{2} / 2} d z / \sqrt{2 \pi}$ and the brackets denote an average over the explicit patterns. The variance of the noise requires recursion relations, not only for the average squared noncondensed overlaps, $\left\langle\left\langle M_{N}^{\mu}(l)^{2}\right\rangle\right\rangle$ and $\left\langle\left\langle M_{N}^{\mu-1}(l)^{2}\right\rangle\right\rangle$, which can be derived in the usual way [27], but also for the correlation of two consecutive overlaps $\left\langle\left\langle M_{N}^{\mu}(l) M_{N}^{\mu-1}(l)\right\rangle\right\rangle$. This generates, in turn, correlations between next-to-consecutive overlaps, $\left\langle\left\langle M_{N}^{\mu}(l) M_{N}^{\mu-2}(l)\right\rangle\right\rangle$ and so on, which requires keeping track of a general form,

$$
\begin{aligned}
C_{n}^{2}(l)= & \sum_{\mu=c+1}^{p}\left\langle\left\langle[ b M _ { N } ^ { \mu } ( l ) + ( 1 - b ) M _ { N } ^ { \mu - 1 } ( l ) ] \cdot \left[ b M_{N}^{\mu-n}(l)\right.\right.\right. \\
& \left.\left.\left.+(1-b) M_{N}^{\mu-n-1}(l)\right]\right\rangle\right\rangle,
\end{aligned}
$$

leading altogether to $p-c+1$ recursion relations,

$$
\Delta^{2}(l+1)=\widetilde{b}^{2}\left(\alpha+\beta^{2} I^{2} \Delta^{2}(l)\right)+2 b(1-b) \beta^{2} I^{2} C_{1}^{2}(l),
$$

$$
C_{1}^{2}(l+1)=\widetilde{b}^{2} \beta^{2} I^{2} C_{1}^{2}(l)+b(1-b)\left[\alpha+\beta^{2} I^{2}\left(\Delta^{2}(l)+C_{2}^{2}(l)\right)\right],
$$

$$
C_{n}^{2}(l+1)=\widetilde{b}^{2} \beta^{2} I^{2} C_{n}^{2}(l)+b(1-b) \beta^{2} I^{2}\left(C_{n-1}^{2}(l)+C_{n+1}^{2}(l)\right),
$$

for $n=2, \ldots, p-c-1$ and $C_{p-c}=\Delta$, where

$$
\begin{gathered}
\tilde{b}^{2}=b^{2}+(1-b)^{2}, \\
I(l)=1-q(l) .
\end{gathered}
$$

Here, $q(l)=\left\langle\langle\sigma(l)\rangle^{2}\right\rangle$ is the spin-glass order parameter given by

$$
q(l)=\left\langle\int D z \tanh ^{2}\{\beta[\boldsymbol{\xi}(l+1) \cdot \boldsymbol{A} \boldsymbol{m}(l)+\Delta(l) z]\}\right\rangle .
$$

Note that the Gaussian noise is symmetric under the change $b \rightarrow(1-b)$ and that in the case where either $b$ is one or zero, that is, for purely Hebbian or sequential noise, respectively, the recursion relation for the variance reduces to the simple form

$$
\Delta^{2}(l+1)=\alpha+\beta^{2} I^{2}(l) \Delta^{2}(l) .
$$

Otherwise, one has to face the full set of recursion relations that may become quite a task for an asymptotically large $p$. Indeed, the system of relations may not form a closed and finite set, and there is no guarantee that this is not, in general, the case. Fortunately, by working out numerically the equations for the dynamics in all the cases we were interested in this work, we found that the set of recursion relations is practically finite and one proceeds as follows.

Given an initial overlap $\boldsymbol{m}(1)=\left\{m^{\mu}(1)\right\}, \mu=1, \ldots, c$, on the first layer, and taking $\Delta^{2}(1)=C_{n}^{2}(1)=\alpha$, for all $n$, Eqs. (11)-(17) describe the dynamics of the network and its stationary states. The latter are given by the fixed-point solutions $\boldsymbol{m}^{*}=\boldsymbol{m}(l+1)=\boldsymbol{m}(l), \Delta^{*}=\Delta(l+1)=\Delta(l)$, and $C_{n}^{*}=C_{n}(l$ $+1)=C_{n}(l)$, for each $n=1, \ldots, p-c-1$. Expressing $C_{1}^{*}$ in terms of all higher $C_{n}^{*}$ s yields $\left(\Delta^{*}\right)^{2} \propto \alpha$, and in the case of $b$ either one or zero this reduces to the simple form

$$
\left(\Delta^{*}\right)^{2}=\frac{\alpha}{1-\beta^{2}(1-q)^{2}},
$$

where $q$ is the fixed-point value of $q(l)$.

There may also exist other solutions, as nonstationary states, as will be seen in the next section. Solving for $T=0$, 
searching for fixed-point solutions and for nonstationary states, one finds that only a small number of the $C_{n}^{*}$ 's on the $(p-c)$-cycle of noncondensed overlaps are clearly nonzero, and all others vanish. The ones that survive become smaller with increasing $n$, indicating the vanishing of the averaged overlaps $\left\langle\left\langle M_{N}^{\mu}(l) M_{N}^{\mu-n}(l)\right\rangle\right\rangle$.

In the case of the finite loading of patterns, where $\alpha=0$, the variance of the noise vanishes and the equations for the overlaps and the spin-glass order parameter become disconnected, and we recover the equations for the overlaps found in previous work [21].

\section{RESULTS}

We show and discuss next our results and use the solutions for finite loading as a guide. We are mainly interested in the network performance in the case of training with a macroscopic number of patterns and on the impact of stochastic noise on the dynamics and the stationary states. Among the important features are the critical loading level, the relative size of the regions with meaningful information processing, the presence of stable symmetric mixture states, and quasiperiodic nonstationary solutions. We also want to find out the specific dependence, if any, of these properties on the form of the stochastic noise, and we restrict ourselves to Hebbian or Hebbian plus sequential noise.

The phase diagrams discussed below are obtained from the dynamical equations for the macroscopic order parameters, and they depend naturally on initial conditions. Quite different sets of these conditions may reflect distinct basins of attraction of the stationary states of the model (specified by a given pair of values of $\nu$ and $b$ ) for given $T$ and $\alpha$. It will be seen that nonstationary states can be reached from certain initial overlaps.

We concentrate on a small number of macroscopic condensed overlaps, specifically $c=3$ and $c=4$, since the results depend on whether $c$ is even or odd, with the remaining $p$ $-c$ patterns as noise in the case of extensive loading. This already illustrates the typical results. For specific results on higher values of $c$ and the differences between an even and odd number of patterns in the case of finite loading, we refer the reader to earlier work [21]. Some of these results will be used below to infer the changes that one may expect for extensive loading.

\section{A. Finite loading}

First we reconsider the solutions for finite loading, where $\alpha=0$, in order to show the phases that appear, the periodic features of the cyclic phase and the quasiperiodic solutions in the region of unstable fixed points.

The $(T, \nu)$ phase diagram of stationary solutions for $\alpha$ $=0$ and $c=4$ is shown in Fig. 1 for the best initial overlaps that favor single-pattern reconstruction (Hopfield or Hopfield-like solutions, see later). There is a paramagnetic phase $(P)$ where $\boldsymbol{m}^{*}=0$ and $q=0$ above $T=1$. This is a line of continuous bifurcations to a phase of stable symmetric fixedpoint solutions (S), $m_{1}^{*}=m_{2}^{*}=m_{3}^{*}=m_{4}^{*} \neq 0$, that ends to the left at a boundary (dashed line) obtained analytically, beyond

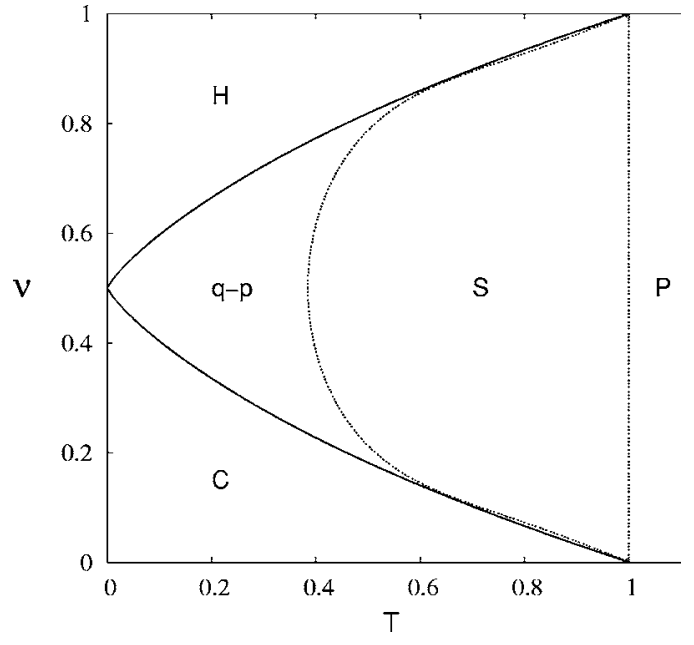

FIG. 1. Phase diagram for $c=4$ condensed patterns for finite loading $(\alpha=0)$, with initial overlaps $m_{1}=1, m_{2}=m_{3}=m_{4}=0$. Hopfield-like $(\mathrm{H})$, symmetric $(\mathrm{S})$, cyclic $(\mathrm{C})$, paramagnetic $(\mathrm{P})$ phases, and region of quasi-periodic (q-p) solutions. Solid and dashed lines indicate first-order and continuous transitions, respectively, here and below.

which the symmetric states become unstable. This is a region of nonstationary quasiperiodic solutions marked q-p, as will be seen later, that is included with the warning that it is not a true part of the phase diagram of stationary states. The same applies to all other phase diagrams in this paper where the q-p solutions are shown. It is worth noting that the symmetric states are the only stable states in the $S$ region due to the stabilizing effect of the static condensed patterns in the learning rule and that this is a characteristic feature of the model, as discussed in Sec. II. Note that the symmetric states are enhanced by synaptic noise.

Eventual alterations of the learning rule aimed at eliminating what has been termed as "spurious" (here not at all) symmetric mixture states, say, by means of the introduction of a bias in the distribution of the patterns [28], would leave nothing but the nonstationary q-p states over a large region of the phase diagram.

The upper region of the phase diagram is a phase of stable Hopfield-like fixed-point solutions $(\mathrm{H})$, with the four components $m_{1}^{*}, \ldots, m_{4}^{*}$ not all equal and different from zero, except at $T=0$, where one component may be one and the other ones zero. Using this zero $T$ result as an initial solution, we construct by numerical iteration of the overlaps the upper and lower phase boundaries indicated by solid lines. All other initial conditions yield phase boundaries above the upper or below the lower line and larger regions of q-p solutions. Those lines are boundaries of first-order transitions for all values of $\nu$, except $\nu=1$ or zero. The first-order transition is to the region of $\mathrm{q}-\mathrm{p}$ solutions with remanent finite overlap components. The location of the transition for $c=4$ is, as expected, close to that for higher $c$ obtained in Ref. [21]. We also find, as expected, larger discontinuities at the first-order transition and shorter remanent overlaps in the q-p region, with our smaller number of condensed patterns than in that reference.

The lower region of the phase diagram is the phase of period-4 stable stationary cyclic solutions (C). These are so- 


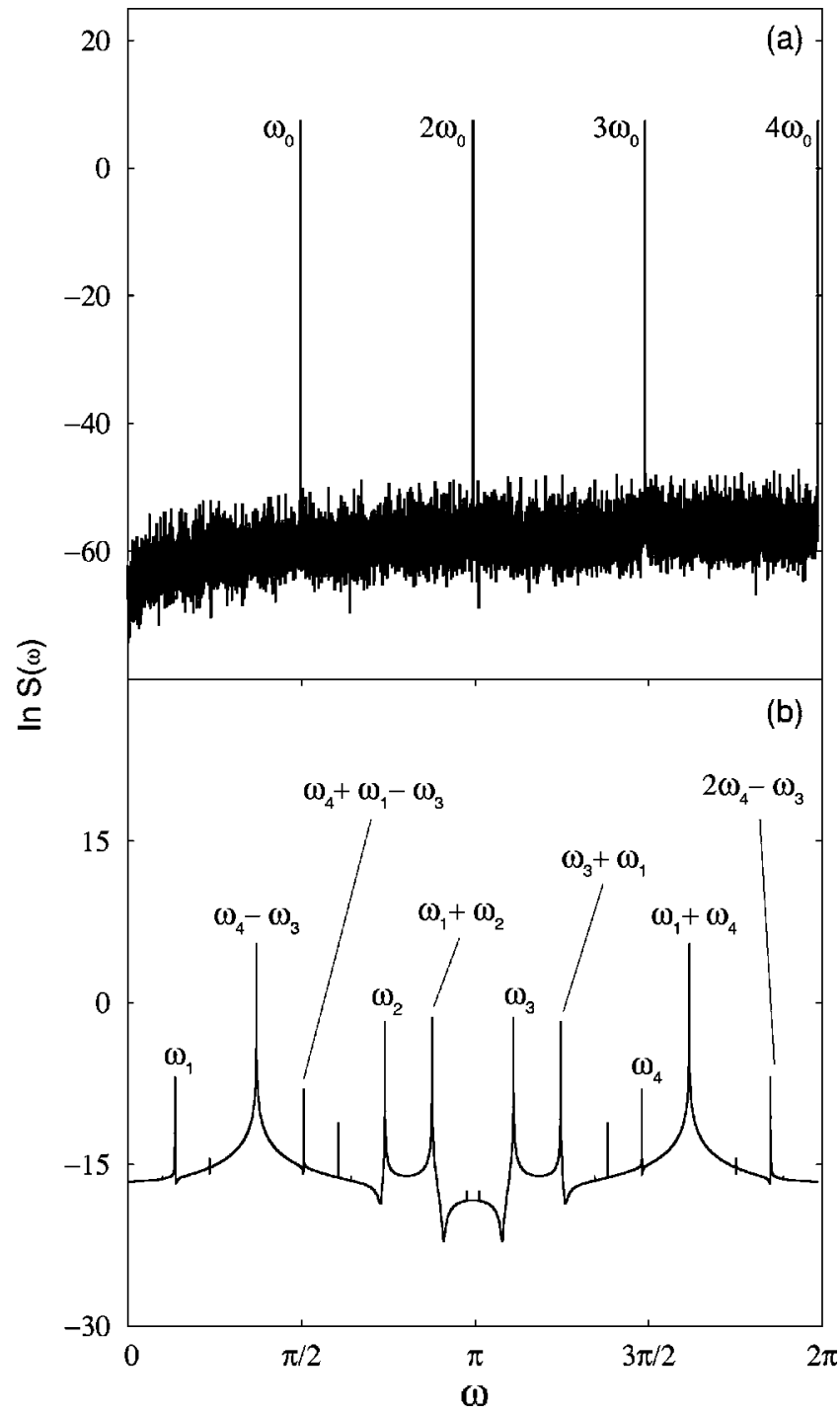

FIG. 2. Power spectrum $S(\omega)$, for $c=4$, and $\alpha=0$. (a) Periodic 4-cycle for $\nu=0.1$ and $T=0.15$ in the C phase, with $\omega_{0}=1.570796$ and its three harmonics. (b) Nonstationary quasiperiodic (q-p) states for $\nu=0.30$ and $T=0.35$ in terms of four basic frequencies.

lutions in which $m_{i}(l+4)=m_{i}(l) \neq 0$ for $i=1, \ldots, 4$, with one overlap close to 1 and the other ones near 0 in each time step after a transient. This fact characterizes sequence processing in which the network makes a transition from one pattern to the next at each layer. The upper and lower first-order phase boundaries appear symmetrically in the phase diagram due to the $\nu /(1-\nu)$ duality, and both are equally sensitive to initial values for the overlaps.

The nature of the cyclic and quasiperiodic solutions for $\alpha=0$ and $c=4$ is best illustrated by the power spectra shown in Figs. 2(a) and 2(b), respectively. The first one is for a typical $\nu=0.1$ and $T=0.15$, within the phase of cyclic states, and the other one is for a typical $\nu=0.30$ and $T=0.35$ in the region of nonstationary states. The power spectrum $S(\omega)$, where $\omega$ is the frequency conjugate to the layer index (a discrete time) may be defined in extension to the case of a continuous time [29], as

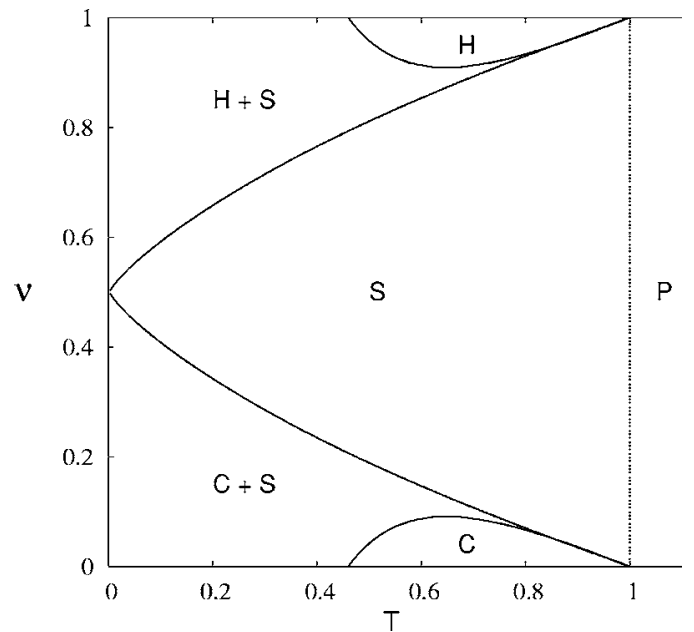

FIG. 3. Phase diagram for $c=3$ condensed patterns for $\alpha=0$ with initial overlaps $m_{1}=1, m_{2}=m_{3}=0$ and phases described in the text.

$$
S(\omega)=(\text { const }) \lim _{L \rightarrow \infty} \frac{1}{L}\left|\sum_{l=0}^{L} e^{i \omega l} m_{1}(l)\right|^{2},
$$

where $m_{1}(l)$ is any one of the components of the overlaps.

The spectrum in Fig. 2(a) clearly exhibits a period-4 solution. In contrast, the spectrum of nonstationary solutions shown in Fig. 2(b) indicates quasiperiodic solutions. These are solutions characterized by main frequencies that are linear combinations of four recognizable basic frequencies.

Consider next the $(T, \nu)$ phase diagram of stable stationary states for $\alpha=0$ and $c=3$, again with initial conditions $m_{1}=1, m_{2}=m_{3}=0$ that favor single-pattern reconstruction, shown in Fig. 3. The symmetric states are now stable over the whole diagram, up to $T=1$, except for an upper and a symmetrically placed lower region of unstable states, where Hopfield-like (H) and cyclic (C) solutions, respectively as indicated, are the only stable solutions. The stability of the symmetric solutions for all $\nu$ at low $T$ is a characteristic feature of the phase diagrams for an odd number of condensed patterns [21]. There is also a pair of tiny q-p solutions, that do not appear on the scale of the figure, between the exclusive $\mathrm{H}$ and $\mathrm{C}$ regions and the exclusive $\mathrm{S}$ phase for high $T$. The $\mathrm{H}$ or $\mathrm{C}$ solutions are stable everywhere above or below the upper or the lower solid lines, respectively. The symmetric states compete for stability with Hopfield-like or cyclic solutions in the regions $H+S$ or $C+S$, depending on initial values of the overlaps. The symmetric states in the $S$ phase are, again, the only stable states in the intermediate region between the two first-order transitions. Other initial more symmetric overlaps yield, again, smaller $\mathrm{H}$ and $\mathrm{C}$ phases and pairs of q-p solutions between the $\mathrm{S}$ and the $\mathrm{H}$ and $\mathrm{C}$ phases.

\section{B. Extensive loading}

For extensive loading, with $\alpha \neq 0$, one has to solve the full set of Eqs. (11)-(17). We still expect to have three ordered phases: $\mathrm{H}$ (Hopfield-like), S, and C, with specific $\boldsymbol{m}^{*} \neq 0$ and $q \neq 0$. Also, a disordered spin-glass phase (SG) should ap- 


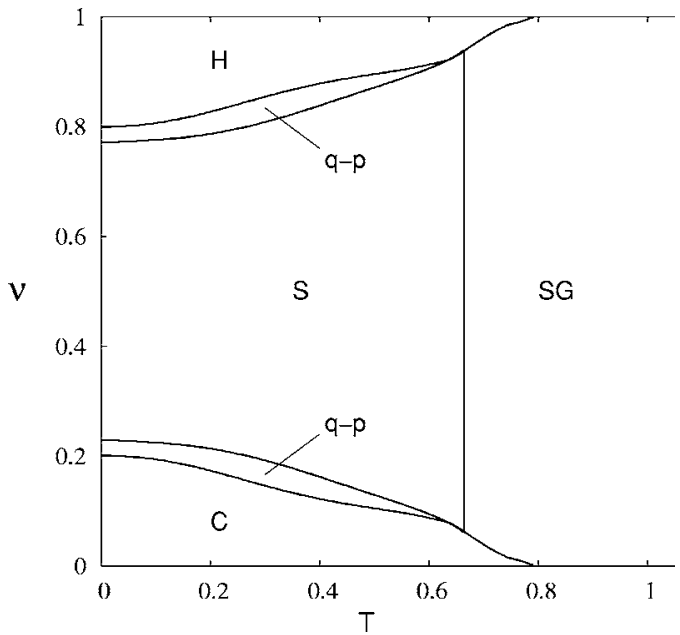

FIG. 4. Phase diagram for $c=4$, extensive loading $(\alpha=0.008)$ and Hebbian noise, with initial overlaps $m_{1}=0.21, m_{2}=m_{3}=m_{4}$ $=0.20$. The spin-glass $(\mathrm{SG})$ phase as indicated.

pear with $\boldsymbol{m}^{*}=0$ and $q \neq 0$ at the end of the ordered phases, and this phase should survive for all higher $T$. The latter is a property of the layered network. All the transitions are now expected to be discontinuous and all the phase boundaries have to be obtained numerically.

One may expect the $\nu /(1-\nu)$ duality also to hold for nonzero $\alpha$. Indeed, the Gaussian noise due to the noncondensed patterns does not change neither under the set of linear transformations that keep the probability distribution of the condensed patterns invariant nor under the permutation index matrix $S=\left\{S_{\mu \rho}\right\}$. These are the two basic transformations that lead to a one-to-one correspondence between every state in the upper part $(\nu>1 / 2)$ in the $(T, \nu)$ phase diagram and a state in the lower part [21]. The duality serves to check the symmetry between the numerically constructed phase boundaries.

The new feature of the $(T, \nu)$ phase diagrams for both $c$ $=3$ and $c=4$, not shown here, that are reached from Hopfield-type initial overlaps that favor single-pattern reconstruction, $m_{1}=1, m_{2}=m_{3}=0$ and $m_{1}=1, m_{2}=m_{3}=m_{4}=0$, respectively, is that the symmetric states become stable at low temperatures, including $T=0$, within a finite range of intermediate values of $\nu$ as soon as $\alpha$ is nonzero. These states remain as the only stable states in that region, and there are $\mathrm{H}$ and $\mathrm{C}$ phases for large and small values of $\nu$, respectively, and a SG phase at high $T$. The phase boundaries to the $\mathrm{H}$ and $\mathrm{C}$ phases are nearly the same for both $c=3$ and $c=4$, and there appear no q-p solutions. The resulting phase diagrams are very similar for either a Hebbian noise or full (Hebbian plus sequential) noise with $b=\nu$, the main difference being that the symmetric phase becomes somewhat enlarged at high $T$ in the latter case.

Other more symmetric initial overlaps, which favor symmetric mixture states, lead to somewhat different results for $c=3$ and $c=4$. For the latter, initial overlaps $m_{1}=0.21, m_{2}$ $=m_{3}=m_{4}=0.20$ reduce the size of the $\mathrm{H}$ and $\mathrm{C}$ regions and lead now to intermediate regions of q-p solutions, as shown in Fig. 4 for $\alpha=0.008$ and Hebbian noise. A very similar diagram is obtained in the case of full noise with $b=\nu$, and

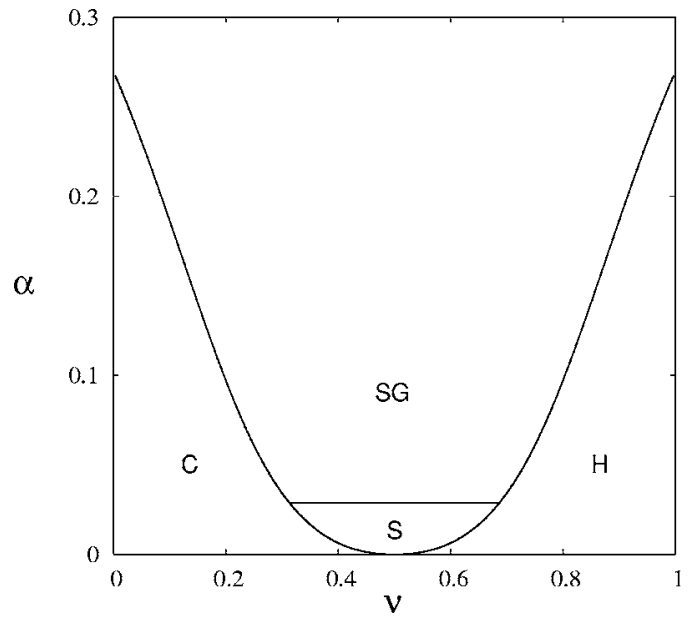

FIG. 5. Phase diagram for $c=4$, extensive loading at $T=0$, and Hebbian noise, with initial overlaps $m_{1}=1, m_{2}=m_{3}=m_{4}=0$.

an analysis of the power spectrum confirms the nature of the $\mathrm{q}-\mathrm{p}$ states as nonstationary quasiperiodic solutions. In contrast, with initial overlaps $m_{1}=0.21, m_{2}=m_{3}=0.20$, in the case of $c=3$, the symmetric states become the only stable states at low $T$ for all values of $\nu$. Regions of stable Hopfield-like and cyclic states appear at higher $T$ and, again, there appear nonstationary q-p solutions between these phases and the $S$ phase.

All components of the overlap in the $\mathrm{H}$ phase are different from zero for nonzero $\alpha$ at $T \neq 0$, although some of them may be small. In contrast, some components may be zero when $T=0$, as in the case of finite loading [21]. Also, for nonzero $\alpha$, the stable states in the $\mathrm{C}$ phase are still period-4 cyclic solutions. We checked this explicitly for $\alpha=0.008$ and values for $\nu$ and $T$ within that phase.

To see the overall role of extensive loading and to find the critical storage ratio $\alpha_{c}$ for a given superposition of Hebbian and sequential learning, we consider next the $(\alpha, \nu)$ phase diagram of stable states at $T=0$ for $c=4$, shown in Fig. 5, for Hebbian noise and initial overlaps $m_{1}=1, m_{2}=m_{3}=m_{4}=0$ that favor single-pattern reconstruction. Note that the critical storage ratio for pure Hebbian learning, that is for $\nu=1$, is $\alpha_{c}=0.269$, in accordance with the known result for the layered network model [27]. Due to the $\nu /(1-\nu)$ duality, it is also seen to be the critical ratio for pure sequential learning of the condensed patterns, as it should be, since a pure sequential noise is a Hebbian noise. The critical storage ratio $\alpha_{c}$ for the retrieval of Hopfield-like or cyclic states is given by a point on the phase boundaries where the $\mathrm{H}$ or $\mathrm{C}$ phases end. In order to check our results obtained from numerical iterations, we performed numerical simulations to locate a few points on the first-order transitions between the ordered phases at $T=0$, and we obtained results in good agreement. As will be seen later (cf. Fig. 7), somewhat different results are obtained in the case of full noise. Also, more symmetric initial conditions, say $m_{1}=0.21, m_{2}=m_{3}=m_{4}=0.20$, lead to further q-p solutions.

We consider next the $(\alpha, \nu)$ phase diagram of stable states at $T=0$ for $c=3$ and Hebbian noise. For the initial overlaps $m_{1}=1, m_{2}=m_{3}=0$ one obtains a similar diagram to that 


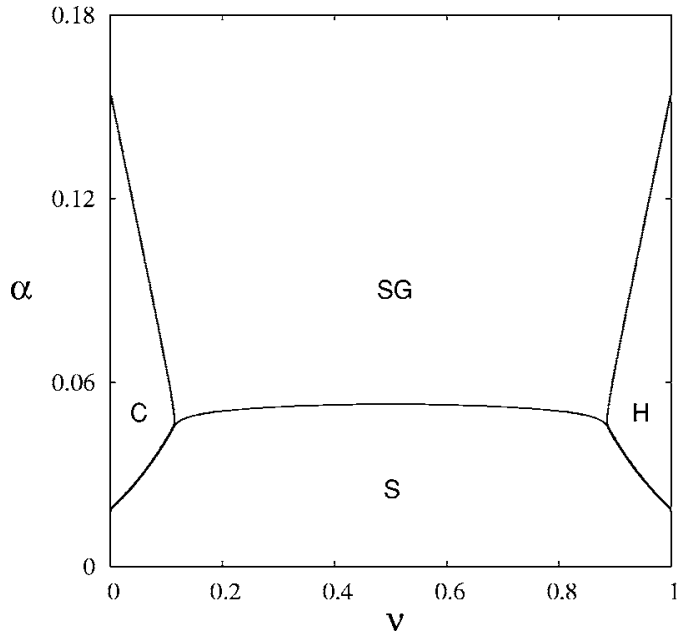

FIG. 6. Phase diagram for $c=3$, extensive loading at $T=0$ and Hebbian noise, with initial overlaps $m_{1}=0.4, m_{2}=0.3, m_{3}=0.2$.

shown in Fig. 5 for $c=4$. The first-order transitions between the $\mathrm{H}$ and the $\mathrm{C}$ phase with the SG phase are close to the case of $c=4$. Instead, for more symmetric initial overlaps, say $m_{1}=0.4, m_{2}=0.3, m_{3}=0.2$, one finds quite a different phase diagram with a symmetric phase almost everywhere below a transition to the SG phase and largely suppressed stable Hopfield-like and cyclic states, as shown in Fig. 6. A similar diagram, except for an enlarged $\mathrm{S}$ phase, is obtained in the case of full noise with $b=\nu$.

We look now at the effects of Hebbian plus sequential noise for $c=4$ and finite $\alpha$ at $T=0$. We do this again for $b$ $=\nu$ and consider also the case of a matrix in which the high components have equally favored Hebbian and sequential parts, in order to explore the symmetry of the model around $b=0.5$, independently of $\nu$. In each case we consider two kinds of initial conditions: one favoring single pattern and cycle retrieval, with $m_{1}=1, m_{2}=m_{3}=m_{4}=0$, and another more symmetric with $m_{1}=0.21, m_{2}=m_{3}=m_{4}=0.20$.

The results for $b=\nu$ and $b=0.5$ for the first initial condition are shown in Figs. 7(a) and 7(b), respectively. In both cases there is now a finite region of nonstationary q-p solutions below the phase of stable symmetric states, that was not present for purely Hebbian noise. This is a feature of complete (Hebbian plus sequential) noise, and in the case of $b$ $=0.5$ there is also a considerable improvement of the critical $\alpha_{c}$ for Hopfield-like and cyclic retrieval. Indeed, we find the quite higher $\alpha_{c}=0.6438$ shown in Fig. 7(b). We also checked with other choices for $b$ and found that $b=0.5$ seems to be the optimal case.

Coming back to the more symmetric initial condition for $c=4$, one finds quite larger regions of stable symmetric states and of nonstationary q-p solutions and particularly smaller regions of $H$ and $C$ phases for both $b=\nu$ and for $b=0.5$.

\section{DISCUSSION AND CONCLUSIONS}

We studied in this work the dynamics of the competition between pattern reconstruction and asymmetric sequence processing in an exactly solvable feed-forward layered neu-

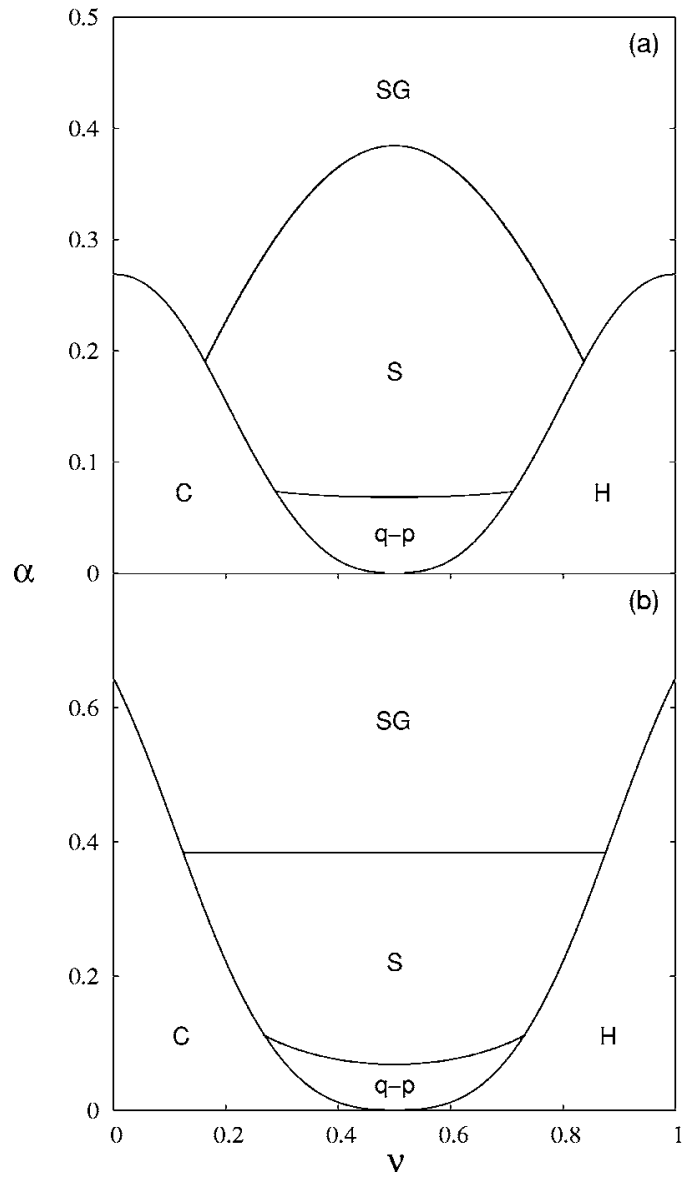

FIG. 7. Phase diagram for $c=4$, extensive loading at $T=0$, and Hebbian plus sequential noise with initial overlaps $m_{1}=1, m_{2}=m_{3}$ $=m_{4}=0$, for (a) $b=\nu$ and (b) $b=0.5$.

ral network model for both finite and for extensive loading of patterns. The strictly feed-forward nature of the network makes it an ideal system to study the dynamics as a discretetime evolution. Given the initial overlaps as inputs on the sites of the first layer, the dynamics is generated by random local fields at every site on the next layer, which depend on the synaptic interaction between units on the two layers. In turn, the local fields determine the probability of updating of units at the sites of that layer. The asymmetry of the sequence processing in this work refers to the sequential part of the learning rule that consists of a synaptic interaction connecting a pattern on a layer, with the consecutive pattern in the sequence on the next layer. A symmetric sequence processing, not considered in this work, would involve an additional synaptic interaction of the same strength connecting a pattern on a layer with the previous pattern in the sequence on the next layer. We come back to this issue below.

The model studied here is based on the superposition of a Hebbian and a sequential learning rule for a finite cycle of condensed patterns and a stochastic noise due to a previously learned macroscopic set of either single patterns that follow a Hebbian rule or a superposition of sequential patterns in a cycle with single patterns. The superposition we consider is suitable for the study of the competition between pattern reconstruction and asymmetric sequence processing. We 
were especially interested in the effects of finite synaptic noise $(\alpha \neq 0)$ on the performance phase diagrams that appear in the case of extensive loading of patterns. Naively, one may expect that the form of the noise does not make a great difference, as we found in most of this work, but this is not always the case.

New dynamic equations for the overlaps and for the variance of the Gaussian noise were obtained in the form of discrete-time recursion relations for the extensive loading of patterns. In the case of a Hebbian plus sequential learning rule with a macroscopic number of patterns $p=\alpha N$, the variance of the noise depends on a macroscopic number of correlations between overlaps with noncondensed patterns, $\left\langle\left\langle M_{N}^{\mu}(l) M_{N}^{\rho}(l)\right\rangle\right\rangle$. All these correlations could be relevant for the dynamics but when it comes to the fixed points (and stationary cycles) only a small number of them survives, making the sequence processing a tractable and solvable problem, at least in the relevant cases we considered in this work. In all the cases we studied the correlations between overlaps with noncondensed patterns formed, practically, a finite and tractable set.

Explicit phase diagrams of stable states and regions of nonstationary solutions were obtained in this work that show the effects of stochastic noise due to a macroscopic number of learned patterns, and different behaviors may be obtained depending on a variety of relevant parameters. We also considered, briefly, the optimal case where $b=0.5$ in the synapses that generate the noise. The reason why this yields a larger $\alpha_{c}$ for $\nu=1$ or zero is that the symmetric phase is considerably enhanced toward extreme values of $\nu$ combined with the fact that Hopfield-like retrieval in this model is, in general, more robust to stochastic noise than the stable symmetric states for small or large $\nu$.

Some general conclusions that may be drawn from the phase diagrams are the following. First, as expected, the retrieval quality of Hopfield-like and cyclic states is gradually reduced with an increase in the storage ratio $\alpha$. Less obvious, there is also a reduction in nearly single-pattern retrieval or in cycle retrieval as $\nu$ or $1-\nu$ are decreased, respectively. The change may be either to a symmetric state or to a spinglass state. Eventually, depending on the form of the noise due to the previously learned patterns, the transition could be to nonstationary quasiperiodic states. The symmetric states appear as a locking of the sequential transitions by the static Hebbian part of the learning rule.

A further result of our work is that, for a Hopfield-type initial condition $m_{1}=1, m_{2}=m_{3}=m_{4}=0$, the first-order phase boundaries in the $(T, \nu)$ phase diagrams where the Hopfieldlike phase ends is practically the same for small $\alpha$ and either Hebbian or complete noise, for both $c=4$ and for $c=3$. That is also the case for the $(\alpha, \nu)$ phase diagrams at $T=0$.
Turning now to predictions for somewhat larger, finite values of $c$, either even or odd for Hopfield-type initial conditions, we expect that the nonstationary solutions that appear in the finite loading case for small $T$ [21] should disappear as soon as $\alpha$ becomes nonzero, allowing for the presence of a symmetric phase down to $T=0$, leaving a phase diagram with essentially no q-p solutions.

It may be pointed out that there is a similarity between our $(\alpha, \nu)$ phase diagrams at $T=0$, with initial Hopfield-type overlaps, and the corresponding phase diagram for pattern reconstruction and symmetric sequence processing referred to above obtained by means of statistical mechanics [18]. The case of pure sequential processing is not considered in that work and, instead of a single-pattern retrieval region found there, we have here a Hopfield-like region (a difference already noted in the context of finite loading [21]). The boundaries of either of these regions have a similar dependence with the ratio $\nu /(1-\nu)$. Moreover, there are similar regions of symmetric phases and, in contrast to our work, there is also a region of correlated states.

Based on the experience with the feed-forward layered network to process information with a Hebbian learning rule, one may expect qualitatively similar results to those obtained here to apply for a recurrent network with a Hebbian-plussequential learning rule. Most of the work reported here deals with the dynamics near stationary states. Since our recursion relations for the overlaps and for the noise are general for the layered network model, they may also be employed to study the transients of the dynamics, in particular, to find out what makes the correlations between distant (in pattern space) high components of the overlaps vanish. One may also study the slowing down of the dynamics due to the macroscopic number of noncondensed patterns. Work along some of these lines is currently in progress.

As an extension of this work one may study the performance of this model with a number of sequences. There is recent work only with sequential learning, for an infinite number of limit cycles [26]. We point out that there is renewed interest in sequence learning from a biological point of view [30] and that asymmetric networks have been found to be computationally superior [31].

\section{ACKNOWLEDGMENTS}

The work of one of the authors (WKT) was financially supported, in part, by CNPq (Conselho Nacional de Desenvolvimento Científico e Tecnológico), Brazil. Grants from CNPq and FAPERGS (Fundação de Amparo à Pesquisa do Estado de Rio Grande do Sul), Brazil, to the same author are gratefully acknowledged. F. L. Metz acknowledges a fellowship from CNPq.
[1] J. J. Hopfield, Proc. Natl. Acad. Sci. U.S.A. 79, 2554 (1982); 81, 3088 (1984).

[2] H. Sompolinsky and I. Kanter, Phys. Rev. Lett. 57, 2861 (1986).
[3] S. Amari and K. Maginu, Neural Networks 1, 63 (1988).

[4] A. V. M. Herz, Z. Li, and J. L. van Hemmen, Phys. Rev. Lett. 66, 1370 (1991).

[5] K. Bauer and U. Krey, Z. Phys. B: Condens. Matter 84, 
131 (1991).

[6] G. Mato and N. Parga, Z. Phys. B: Condens. Matter 84, 483 (1991).

[7] R. Kühn and J. L. van Hemmen, Models of Neural Networks I, edited by E. Domany, J. L. van Hemmen, and K. Schulten (Springer-Verlag, Berlin, 1991), p. 213.

[8] H. Rieger, M. Schreckenberg, and J. Zittartz, J. Phys. A 21, L263 (1988); , Z. Phys. B: Condens. Matter 72, 523 (1988).

[9] A. C. C. Coolen and D. Sherrington, Phys. Rev. Lett. 71, 3886 (1993); , Phys. Rev. E 49, 1921 (1994).

[10] S. N. Laughton and A. C. C. Coolen, Phys. Rev. E 51, 2581 (1995).

[11] M. Okada, Neural Networks 9, 1429 (1996).

[12] A. Düring, A. C. C. Coolen, and D. Sherrington, J. Phys. A 31, 8607 (1998).

[13] Y. Miyashita and H. S. Chang, Nature (London) 331, 68 (1988).

[14] Y. Miyashita, Nature (London) 335, 817 (1988).

[15] M. Griniasty, M. V. Tsodyks, and D. J. Amit, Neural Comput. 5, 1 (1993).

[16] N. Brunel, Network Comput. Neural Syst. 5, 449 (1994).

[17] L. F. Cugliandolo, Neural Comput. 6, 220 (1994).

[18] L. F. Cugliandolo and M. V. Tsodyks, J. Phys. A 27,
741 (1994).

[19] T. Uezu, A. Hirano, and M. Okada, J. Phys. Soc. Jpn. 73, 867 (2004).

[20] D. J. Amit, H. Gutfreund, and H. Sompolinsky, Ann. Phys. 173, 30 (1987).

[21] A. C. C. Coolen and D. Sherrington, J. Phys. A 25, 5493 (1992).

[22] W. Whyte, D. Sherrington, and A. C. C. Coolen, J. Phys. A 28, 3421 (1995).

[23] K. Kitano and T. Aoyagi, J. Phys. A 31, L613 (1998).

[24] M. Kawamura and M. Okada, J. Phys. A 35, 253 (2002).

[25] W. K. Theumann, Physica B 328, 1 (2003).

[26] K. Mimura, M. Kawamura, and M. Okada, J. Phys. A 37, 6437 (2004).

[27] E. Domany, W. Kinzel, and R. Meir, J. Phys. A 22, 2081 (1989); R. Meir and E. Domany, Phys. Rev. Lett. 59, 359 (1987).

[28] D. J. Amit, H. Gutfreund, and H. Sompolinsky, Phys. Rev. A 35, 2293 (1987).

[29] J-P. Eckmann and D. Ruelle, Rev. Mod. Phys. 57, 617 (1985).

[30] B. G. Nielsen, Neural Comput. 14, 189 (2003).

[31] Z. Li and P. Dayan, Network Comput. Neural Syst. 10, 59 (1999). 